\title{
Representaciones sociales de la relación familia-escuela desde la formación inicial del profesorado
}

\section{Social Representation of Family-School Relationship from Initial Teacher Training}

\author{
Héctor Cárcamo Vásquez (1) https://orcid.org/0000-0003-2045-343X \\ Jordi Garreta Bochaca (2) https://orcid.org/0000-0002-3434-9441 \\ (1) Universidad del Bío-Bío \\ (2) Universidad de Lleida \\ (Recibido: 7 de agosto de 2017; Aceptado para su publicación: 5 de marzo de 2018)
}

Cómo citar: Cárcamo, H. y Garreta, J. (2020). Representaciones sociales de la relación familia-escuela desde la formación inicial del profesorado. Revista Electrónica de Investigación Educativa, 22, e11, 1-14. https://doi.org/10.24320/redie.2020.22.e11.2406

\section{Resumen}

La investigación expone las representaciones que poseen los futuros profesores de educación básica de una Universidad de la Región de Ñuble (Chile) sobre la familia y los roles parentales que les son asignados en materia educativa. En concordancia con el objetivo, se desarrolla un estudio comprensivo interpretativo utilizando la metodología cualitativa. Dentro de los hallazgos más relevantes se encontró que los roles que los sujetos asignan a las familias se sitúan en dos dimensiones: por una parte, apoyo en el ámbito pedagógico a través de la realización de deberes escolares y, por otra, la inculcación de hábitos que faciliten el establecimiento de un clima en el aula favorable para el aprendizaje.

Palabras clave: Relación familia-escuela, formación inicial docente, representaciones sociales.

\section{Abstract}

The paper presents the representations that future elementary school teachers have in a University of Ñuble region for the family and parental roles assigned to them in education. In line with the objective, a comprehensive interpretive study using qualitative methodology is developed. Among the most important findings we found that roles that subjects assign families are located in two clearly defined dimensions; on one hand, support on the educational field through the completion school duties and, secondly, the inculcation of habits that facilitate the establishment of a classroom climate in favor of learning.

Keywords: Family-school relationship, initial teacher training, social representations.

\section{Introducción}

Diversos autores plantean la importancia que adquiere el establecimiento de una adecuada relación entre las familias y la escuela en el proceso educativo, entre los aspectos los aspectos que señalan destacan que los antecedentes familiares constituyen un elemento central para el éxito escolar, tanto por el capital cultural educativo como por los recursos que ponen a disposición de dicho proceso (Cerletti, 2010; Domina, 2005; Garreta, 2015; Gubbins, 2014; Lareau, 2002; Martos y Martos, 2010; Londoño y Ramírez, 2012; Segovia et al., 2008). Sin lugar a dudas, el involucramiento de las familias en el proceso de escolarización 
de los niños es clave, pero más si se consideran los primeros años de la infancia (Enkvist 2011; Mc Wayne, 2013; Saraiva y Wagner, 2013).

La literatura especializada no sólo circunscribe los beneficios de una fluida relación familia-escuela al ámbito del aprendizaje y la mejora educativa; también destaca la relevancia que posee dicho trato para la democratización del espacio escolar, la formación ciudadana y la densificación del tejido social (Gubbins, 2016), además de que favorece actitudes más positivas de las familias respecto al centro escolar (Grant y Ray, 2013), mejora la probabilidad de que el alumnado mejore actitudes y resultados académicos (Epstein 2004) y, entre otras cuestiones, contribuye de forma significativa a la moral de los docentes, así como al aprendizaje del alumnado y a la satisfacción de los progenitores (Walker y Hoover-Dempsey; 2008). Este reconocimiento pone de manifiesto la relevancia de la relación familia-escuela desde las diferentes aristas que la configuran como problema social, atendiendo a la diversidad de agentes, momentos, espacios e instancias que entran en juego, así como los diferentes ámbitos del fenómeno, por ejemplo los niveles de participación (Palaudàrias, 2017) y los estilos y mecanismos de comunicación (Garreta y Maciá, 2017).

En el contexto de esta investigación, el Ministerio de Educación de Chile (MINEDUC) ha establecido los estándares orientadores para los egresados de la carrera de Pedagogía en educación básica (MINEDuc, 2012). Al respecto, el estándar pedagógico número 8 refiere que el egresado debe estar preparado para atender la diversidad y promover la integración en el aula, cobrando relevancia la relación que establezca el profesorado con el contexto del niño y su familia. Otro instrumento en el cual se aprecia la importancia otorgada a la familia en la escolarización de los niños es la prueba SIMCE $^{1}$, en el cuestionario enviado a la familia se busca explorar los indicadores de desarrollo personal y social propuestos por la Agencia de Calidad de la Educación, por cuanto buscan medir el impacto que tiene la implicación parental en dimensiones del ámbito personal y social del niño (autoestima académica, participación y formación ciudadana, entre otros).

Aunque los instrumentos vigentes a nivel del MINEDUC y la Agencia de Calidad de la Educación aluden a la relevancia de la participación de las familias en la escuela, es en la Política de Participación de las Familias y la Comunidad en Instituciones Educativas (MINEDUC, 2017) donde se explicita la relevancia de promover la participación y el involucramiento de las familias en el proceso de escolarización. No obstante, a pesar de este reconocimiento, los estudios respecto a esta temática son escasos y circunscritos a la escuela, siendo prácticamente inexistentes aquéllos que abordan la problemática de la relación familia-escuela desde la formación inicial docente.

Este artículo atiende a lo que acontece en el contexto de la formación inicial del profesorado de educación básica (primaria) tomando como teoría general las representaciones sociales con énfasis en su dimensión de contenido (Moscovici, 1979). A este respecto, Calixto (2015) afirma que las representaciones sociales revisten gran importancia para el abordaje del fenómeno educativo, ya que "a través de éstas, es posible vincular el conocimiento científico con el conocimiento de la vida cotidiana" (p. 47).

El develamiento de las representaciones sociales respecto a diversos objetos de representación facilita la problematización de los fenómenos y la eventual desnaturalización de acciones reproductivas que atentan contra la transformación social. Un claro ejemplo lo encontramos en Río (2010), quien pone en evidencia la supremacía de la representación que el profesorado posee respecto a las familias y su escasa implicación en el proceso de escolarización: "las familias no quieren participar". No obstante, el autor releva la necesidad de visualizar lecturas alternativas -a modo de complemento- sobre la base de expresiones como: "las familias no saben participar" o "las familias no pueden participar".

Siguiendo lo expuesto por Piña (2013) y Cárcamo (2015), entre otros, aproximarse al mundo de las representaciones respecto al fenómeno educativo es relevante ya que éstas intervienen directamente sobre los modos de actuación de los sujetos en la práctica social, pues tal como sostiene Jodelet (1986, p.

\footnotetext{
1 Sistema de evaluación utilizado por la Agencia de calidad de la Educación con el fin de evaluar resultados de aprendizaje de los establecimientos educacionales de todo el país. Además de las pruebas del área curricular se recoge información relativa a las familias a través de un cuestionario específico.
} 
474) las representaciones sociales corresponden a "modalidades de pensamiento práctico". Así, las representaciones pueden ser entendidas como "sistemas de ideas, concepciones, actitudes y valores, que constituyen principios orientadores de las relaciones cotidianas" (Calixto, 2015, p. 14). Desde esta perspectiva el sentido común adquiere trascendencia; en torno a éste se modelan las formas de hacer y ser en el mundo por parte de colectivos específicos, en este caso estudiantes de la carrera de Pedagogía en educación general básica. ${ }^{2}$

El abordaje de las representaciones sociales permite una aproximación a los sentidos y significados atribuidos por los sujetos a determinados objetos de atención. Dicho entramado de sentidos y significados permite visualizar los elementos que configuran al objeto representado, las fuentes que intervienen en este modelamiento y el proceso que subyace a él. Cabe mencionar que la forma de configurar la representación y la representación en sí misma se fundan en la interacción social (Lacolla, 2015).

Dado que la relación familia-escuela es una de las aristas del fenómeno educativo, estudiar los contenidos, fuentes y agentes que intervienen en la configuración de las representaciones respecto a esta relación se vuelve pertinente y necesario, sobre todo si se considera que la relación familia-escuela varía según la representación que posea el propio profesorado y (para acercarnos a nuestro objeto de estudio) quienes cursan su formación inicial docente. Garreta (2015) y Pascual y Gomila (2012) afirman que la relación (en forma y contenido) que se establece entre las familias y la escuela se modela tempranamente a partir de las representaciones que circulan en el propio proceso de formación inicial del profesorado. A pesar de ello, el estudio de las representaciones sociales en el campo de la formación universitaria inicial docente ha sido escasamente abordado. Autores como Cárcamo-Vásquez y Rodríguez-Garcés (2015) y Pérez (2010) plantean la necesidad de abordar la temática de la relación familia-escuela desde este contexto, de modo tal que haya una reflexión crítica sobre esta arista del fenómeno educativo, cuya presencia en el proceso de formación inicial docente es más bien accesorio. ${ }^{3}$

Al considerar el desarrollo alcanzado por la teoría de las representaciones sociales, resulta adecuado su uso en tanto que teoría general para interpretar los datos, ya que facilita la comprensión en torno a la configuración y reconfiguración del conocimiento producido socialmente (Jodelet, 1986; Moscovici, 1979; Piña 2013). Poniendo en evidencia su vínculo con las imágenes, opiniones, estereotipos y creencias, que los futuros profesores de educación básica tienen sobre la relación familia-escuela. Investigar esta temática desde la teoría de las representaciones sociales contribuirá a problematizar y desnaturalizar diversos aspectos que conducen a la reproducción de modelos relacionales fundados en lógicas participativas de carácter informativo, básicas, tal como lo señala Garreta (2017) y Schilling (2015).

El objetivo de este trabajo es reconocer las representaciones sociales que poseen los alumnos que estudian para ser profesores de educación básica respecto a los roles parentales asignados a las familias en materia educativa.

\section{Método}

Dado el interés por situarnos desde la perspectiva de los agentes, desde el paradigma comprensivo nos apoyamos epistemológicamente en la sociofenomenología, pues el acento está depositado en la forma de construcción social del conocimiento situado y la circulación del mismo. Valorando la subjetividad de los agentes y los procesos derivados de la intersubjetivación (Schutz, 2003). Siguiendo a Toledo (2014), se buscó descubrir y reconstruir la red de sentidos y significados que configuran las representaciones que los estudiantes que cursan la carrera de Pedagogía en educación básica poseen respecto de las familias y su relación con la escuela.

Las preguntas orientadoras del proceso investigativo fueron: ¿cómo representan los futuros profesores de

\footnotetext{
2 En Chile, el nivel de educación básica es de tipo obligatorio y tiene una duración de 6 años, equivalente a la educación primaria de otros contextos.

${ }^{3}$ Dicha situación se constata tras analizar los planes de estudios de las carreras de Pedagogía en educación básica de universidades de las regiones de Ñuble y Bío-Bío.
} 
educación básica la relación familia-escuela?, ¿cuáles son las imágenes que poseen respecto a las familias y la implicación parental educativa?, ¿qué roles significan como relevantes para ser desempeñados por las familias en el proceso de escolarización?

Para estos efectos se utilizó la metodología cualitativa, dado el énfasis otorgado a la subjetividad de los agentes, así como a la circulación de los sentidos y significados atribuidos por éstos a los siguientes objetos de representación: relación familia-escuela, implicación parental en el proceso educativo y rol que deben desempeñar las familias, que se abordan atendiendo a los contenidos que conforman la representación, sus fuentes y sus agentes.

El método seleccionado corresponde al estudio de casos único de alcance descriptivo (Coller, 2005), centrado en los discursos de estudiantes de Pedagogía en educación general básica de la única universidad pública y estatal de la región de Ñuble (zona centro-sur de Chile), una universidad de larga tradición en la formación de profesorado que se desempeña en el nivel básico de educación en establecimientos de la región. En el momento de la investigacion estaban adscritos a la carrera 144 estudiantes, provenientes de establecimientos educacionales distribuidos en las 21 comunas que conforman la región.

El levantamiento de información se desarrolló a través de la entrevista semi-estructurada, pues como afirma Gaínza (2006), su naturaleza dialógica permite acceder a los sentidos y significados que los agentes atribuyen a los objetos de atención. La pauta de entrevista fue sometida a juicio experto para resguardar su pertinencia estructural y de contenido.

El corpus discursivo se conformó por 14 entrevistas a alumnos de la carrera de Pedagogía en educación básica pertenecientes a diferentes cohortes: 10 son mujeres y 4 hombres de 19 a 22 años de edad. El procedimiento para la selección de los sujetos responde al muestro intencional.

Los criterios de inclusión considerados fueron: accesibilidad, heterogeneidad de cohorte de ingreso a la carrera y sexo. La heterogeneidad de cohorte se expresa en la selección de siete estudiantes que cursan el tercer año y siete que cursan el cuarto año, esta diferenciación se explica porque de acuerdo al plan de estudios los estudiantes deben realizar sus prácticas. En el caso de tercer año, efectúan una práctica en establecimientos educacionales rurales; ${ }^{4}$ por su parte, los de cuarto año realizan su práctica profesional. En ambos casos tienen la posibilidad de vincularse con las familias y la comunidad educativa. Respecto a la heterogeneidad de sexo, se intentó acceder a hombres y mujeres, aunque fueron más las mujeres porque la matrícula está más representada por este grupo poblacional. En cuanto al criterio de homogeneidad, éste se manifiesta en tanto que la totalidad de los sujetos son alumnos de la carrera de pedagogía en educación general básica de la universidad estudiada. El trabajo de campo se desarrolló durante el segundo semestre del 2016 y el primer semestre del 2017.

El acceso a los sujetos se realizó a través de la secretaria de la carrera, resguardando el principio de voluntariedad. Asimismo, se atendieron los protocolos éticos mediante el resguardo de la confidencialidad de los sujetos y el uso exclusivo de la información para efectos investigativos.

El análisis de discurso se trabajó a través de la técnica de análisis estructural, pues como sostiene Martinic (2006), es útil para abordar el flujo de significaciones de las representaciones. El procedimiento se desarrolló de la forma siguiente: Tras la transcripción de las entrevistas se procedió con la codificación inicial abierta de tipo vertical. Luego, un análisis temático, a partir del cual se generó una recodificación del material discursivo. La combinación de ambas estrategias permitió generar el proceso de categorización en sus tres etapas (abierta, selectiva y axial) sobre la base del cual se identifican ejes de significación que facilitan el reconocimiento de la regularidad discursiva y la consecuente identificación de elementos constitutivos de las representaciones (ver figura 1). Finalmente, los resultados que se exponen se desprenden de la triangulación teórico-empírica mediante el reconocimiento de ejes de significación y sus respectivos códigos disyuntivos.

\footnotetext{
${ }^{4}$ Esta práctica se da en modalidad de internado. Los estudiantes deben vivir en la escuela durante un mes.
} 


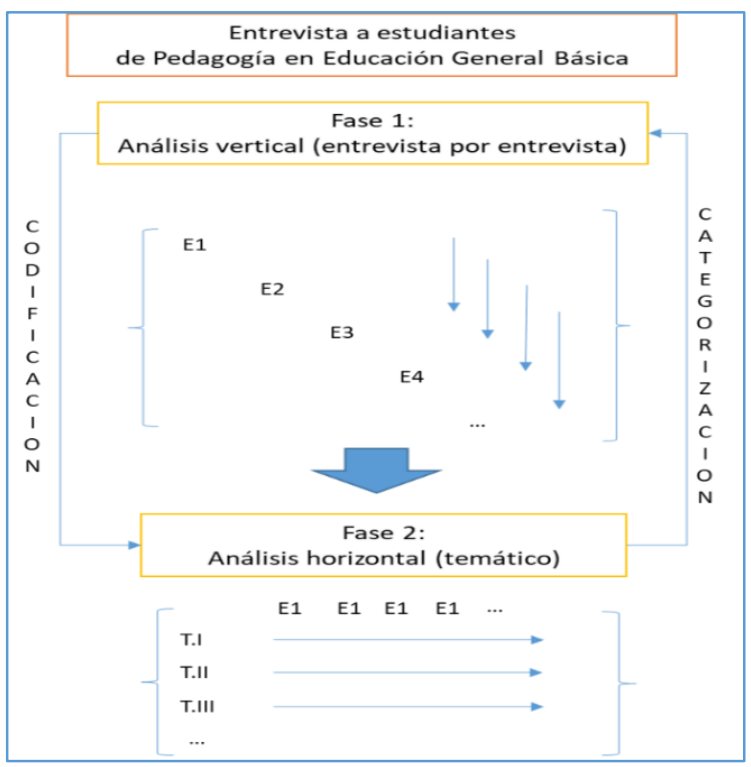

Figura 1. Procedimiento analítico del corpus discursivo

\section{Resultados}

Tras el análisis se pudo constatar que la configuración de la representación en torno a la relación familiaescuela que circula entre los sujetos se forja sobre la base de la asignación de determinados roles parentales en materia educativa, así como por los roles que asignan al profesorado. Dicha representación está fundada en elementos derivados de la propia experiencia de escolarización y la formación inicial docente.

Los resultados se exponen en tres apartados. El primero refiere a la asignación de roles parentales que los sujetos asignan a las familias. El segundo, enfatiza en los roles que los futuros docentes asignan al profesorado. Por último, presentaremos los elementos de mayor relevancia respecto de las formas en que los sujetos representan la relación familia escuela.

\subsection{Asignación de roles parentales}

En los discursos se aprecia una regularidad en cuanto a los roles que los futuros profesores consideran deben ser cumplidos por las familias. Estos roles son conceptualizados, priorizados y asignados por los sujetos sobre la base de la experiencia propia de escolarización en el nivel básico de educación y el actual proceso de formación inicial docente, sin atender a los elementos consignados en los estándares pedagógicos que relevan la necesidad de que el egresado de la carrera de educación básica atienda a la diversidad y promueva la integración en el aula respetando y valorando el acervo cultural familiar de sus alumnos.

Los roles giran en torno a un eje fundamental, el cual se relaciona con la posibilidad de extender el área de acción de la escuela a los espacios no escolares. Se busca la escolarización de los espacios extra escuela, de modo que valoran todas aquellas acciones a complementar el proceso de escolarización que tienen cabida en las escuelas (ver figura 2). El eje de significación corresponde al proceso de escolarización. Se valora todo aquello que dé cuenta de expansión de la escolarización a espacios no escolares, por ejemplo, la vida familiar. 


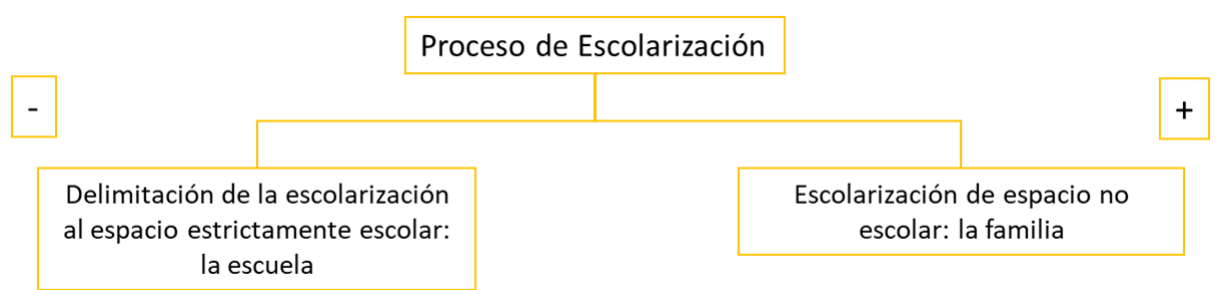

Figura 2. Eje de significación: proceso de escolarización

Los roles que los futuros docentes esperan que desempeñen las familias están asentados en dos ámbitos claramente delimitados, el primero alude a aspectos estrictamente pedagógicos y el otro refiere a la formación de hábitos, aunque siempre como complemento de la dimensión pedagógica. Hábitos que, por cierto, se cree contribuyen al buen desempeño del niño como alumno (figura 3).

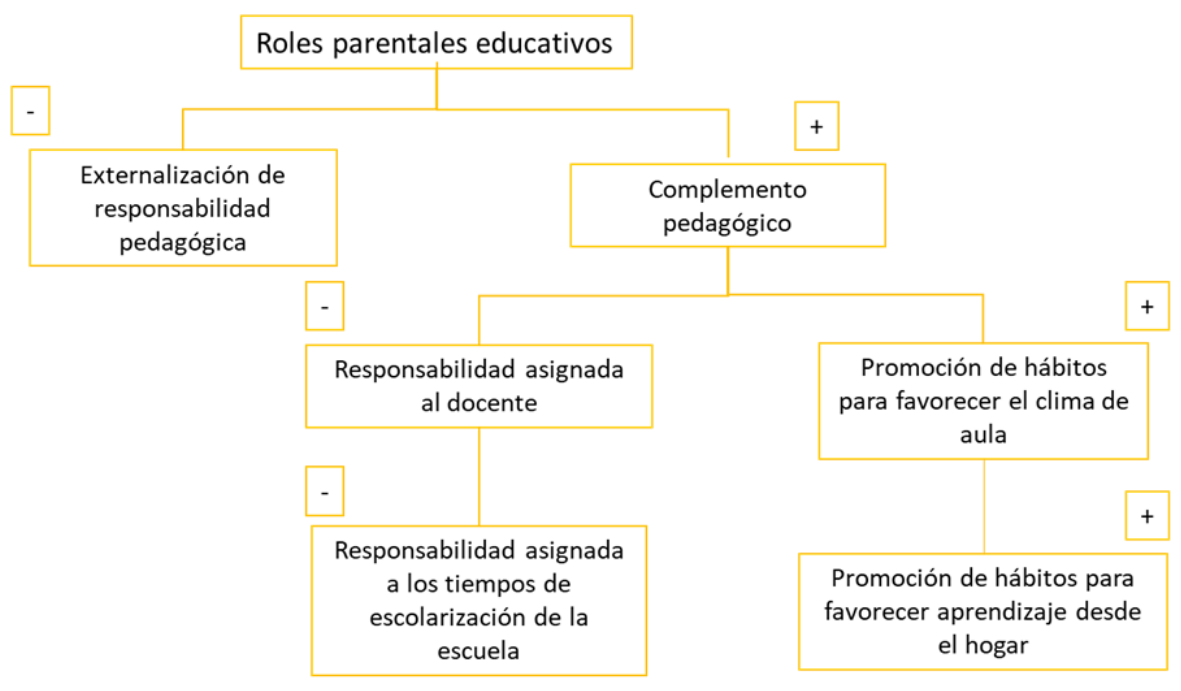

Figura 3. Eje de significación: roles parentales educativos

A este respecto, Ignacio es elocuente al referirse a lo que debe hacer la familia:

Motivar, incentivar. Hacer lo mismo que hace un profesor en el aula, pero con la autoridad que tiene un padre o un hermano, que es muy distinto a la que tiene un profesor. Al menos eso es lo que hacía mi mamá. (Ignacio)

Tal como se aprecia en el extracto precedente, se espera que las familias -ya no sólo madre o padre, sino también hermanos- asuman acciones tendientes a extender figurativamente el tiempo dedicado a la escolarización tomando como referente las demandas generadas por el cuerpo docente, sin reconocer como posibilidad la instalación de lógicas de reciprocidad y dialogicidad destacadas como necesarias en los estándares pedagógicos propuestos por el Ministerio de Educación (MINEDUC, 2012). Pero qué es lo que demanda el cuerpo docente. En primer término, tal como se ha señalado, apoyo en el quehacer pedagógico. Uno de los entrevistados indica lo siguiente:

Idealmente la familia tiene que involucrarse lo más posible en la escuela, obviamente hay una labor que la cumple el profesor por razones obvias porque ellos se prepararon para eso, por ejemplo, están capacitados para hacerlo, para enseñarles algo. Ahí más que intervenir los papás y las familias, deben apoyar reforzando a los niños más que nada en la parte de entrega de conocimiento. (Esteban) 
Los sujetos consideran que desde la casa se debe complementar la experiencia de la escolarización para potenciar aspectos desarrollados en el aula. Aunque también se reconoce la posibilidad de que los padres no estén en condiciones de responder adecuadamente a las exigencias académicas demandadas, ya sea por cambios en los contenidos curriculares, cambios en las estrategias de enseñanza, o bien, por la baja escolaridad de los mismos. No obstante, al reconocer la posibilidad de que las familias no sepan cómo resolver situaciones de contenidos, se les asignan responsabilidades frente al apoyo para la promoción de actitudes que contribuyan al mejor desempeño de los niños (ver figura 3). Al respecto, Fernanda sostiene lo siguiente:

Es entendible que los papás por ejemplo siempre dicen no, es que yo no sé esas materias, no sé cómo ayudar a mi hijo, pero siempre hay formas. Por ejemplo, el simple hecho de revisarle todos los días el cuaderno es una forma de ayudar para que el niño lo haga solo después, aunque independiente de que no sepa la materia. Se supone que las reuniones de apoderados, bueno por lo que he aprendido en la carrera, deberían enseñarte a cómo ayudar a tu hijo, y ahí es el rol del profesor, enseñarle al papá a cómo ayudar a su hijo, no siempre se cumple, pero debería ser así. Entonces, por último, revisarle los cuadernos, ir a las reuniones, ir a conversar en las horas que da el profesor para hablar de su hijo, de cómo está la situación. (Fernanda)

Junto con los aspectos pedagógicos y la promoción de hábitos, aparece otro aspecto, el cual podría considerarse como elemento complementario que sirve de barómetro al docente para evaluar el nivel de compromiso familiar en el desempeño escolar de los niños. Este aspecto refiere a la presencia de los padres y apoderados en las instancias estipuladas por los establecimientos educacionales (figura 4). Los entrevistados aluden a: asistencia a reuniones y asistencia a las entrevistas personales convocadas por el profesor, como señala Fernanda, o instancias que, como sostiene Camila, sirven para una cuestión central, atender al desempeño académico del niño/alumno:

Asistir a reuniones para ver cómo va la situación del niño, si va subiendo sus notas, si va bajando, buscar métodos de ayuda a pesar de que no sepan de las materias, buscar por otra parte a lo mejor un tío o un primo, algo, que sepa de esto y que ayudara al niño. (Camila)

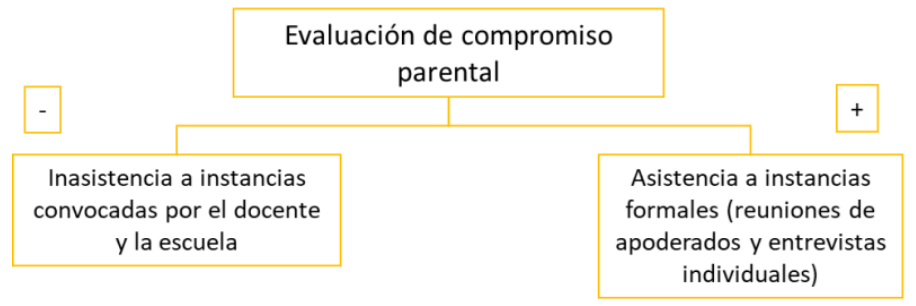

Figura 4. Eje de significación: evaluación del compromiso parental

De acuerdo con los entrevistados son las familias las responsables de buscar estrategias que promuevan un buen desempeño del niño en el contexto escolar. La escuela facilitaría espacios formales, tales como reuniones de apoderados y entrevistas individuales, para transmitir la información que la escuela y el profesor consideran relevante. Así, se proporciona una línea de base a partir de la cual las familias debieran asumir (desde casa) actuar para que los niños cumplan los objetivos trazados por la escuela en la escuela.

\subsection{Rol del profesorado}

La representación respecto del rol que debe desempeñar el profesorado es otro elemento que se constituye en eje de significación de la relación familia-escuela. La imagen que poseen los entrevistados en torno al papel que han de desempeñar los docentes y ellos mismos en un futuro próximo se modela a partir de la distribución de tareas entre la escuela y la familia. Los profesores deben realizar ciertas cosas y las familias otras. 
¿Qué es lo que debe realizar el docente?, a través de los discursos se reconocen dos tipos de elementos. Por una parte los contenidos y por otra las herramientas. Según los sujetos, los docentes son responsables de transmitir a sus alumnos un conjunto de conocimientos plasmados curricularmente según el área disciplinar. Por otra parte, el profesorado debe proporcionar herramientas prácticas que permitan a los alumnos desenvolverse en sociedad de forma adecuada, para que sean -como menciona Loreto- "mejores personas".

Saber que puedo ayudarlos a ser mejores personas, a tratar de formarlos como tal, como personas que van a ser. (Loreto)

La transmisión de conocimientos (teórico de tipo académico) y de herramientas (valóricas y actitudinales) se presentan como dimensiones constitutivas de la persona, una buena persona. A este respecto, Ignacio sostiene:

El profesor te entrega los contenidos y las herramientas, pero por otra parte también va forjando tu..., no sé si decir personalidad, tus puntos fundamentales que te van a ayudar a ser una persona buena en la vida, como la puntualidad, la responsabilidad, la solidaridad. (Ignacio)

Para conseguir este objetivo es fundamental que el profesorado asuma el rol de guía o facilitador de este proceso academicista y moral respecto al alumnado y también de las familias, con el fin de facilitar ajustes en favor del proyecto educativo del establecimiento educacional. Llama la atención que no consideren la variabilidad del nivel cultural de los padres para referir al rol de guía. No son conscientes de que en determinados contextos socioculturales son las familias las que asumen un rol más protagónico en relación al vínculo con el profesorado y la escuela.

¿Cómo se relaciona la representación del rol docente al que refieren los entrevistados con los elementos curriculares de carácter nacional? Considerando las bases curriculares para educación básica vigentes desde el 2013 (MINEDUC, 2013), no deja de ser de interes la configuración de esta representación por su restringido campo de acción. No se considera como opción la articulación de la diversidad de agentes que confluyen en el proceso de escolarización de los niños, no se hace sino relevar la noción de guía de un proceso cuya dirección, contenidos y formas se modelan desde el campo escolar. El énfasis está puesto en una lógica unidireccional, desde la cual el saber experto especializado se legitima a sí mismo.

La figura de guía aparece dentro del discurso de forma paradójica o un tanto sui generis, puesto que lo que se constituye como deseable (el ser guía) no se ajusta con lo que debe hacer en este doble proceso: transmitir. El profesor debe transmitir conocimientos y valores; el profesor debe entregar herramientas y contenidos (figura 5). Al respecto, Camila sostiene:

La mayoría de los profesores que conozco son de esa línea, de entregarte no tan solo contenidos sino herramientas que te sirven en la vida, eso es lo que debemos hacer. (Camila) 


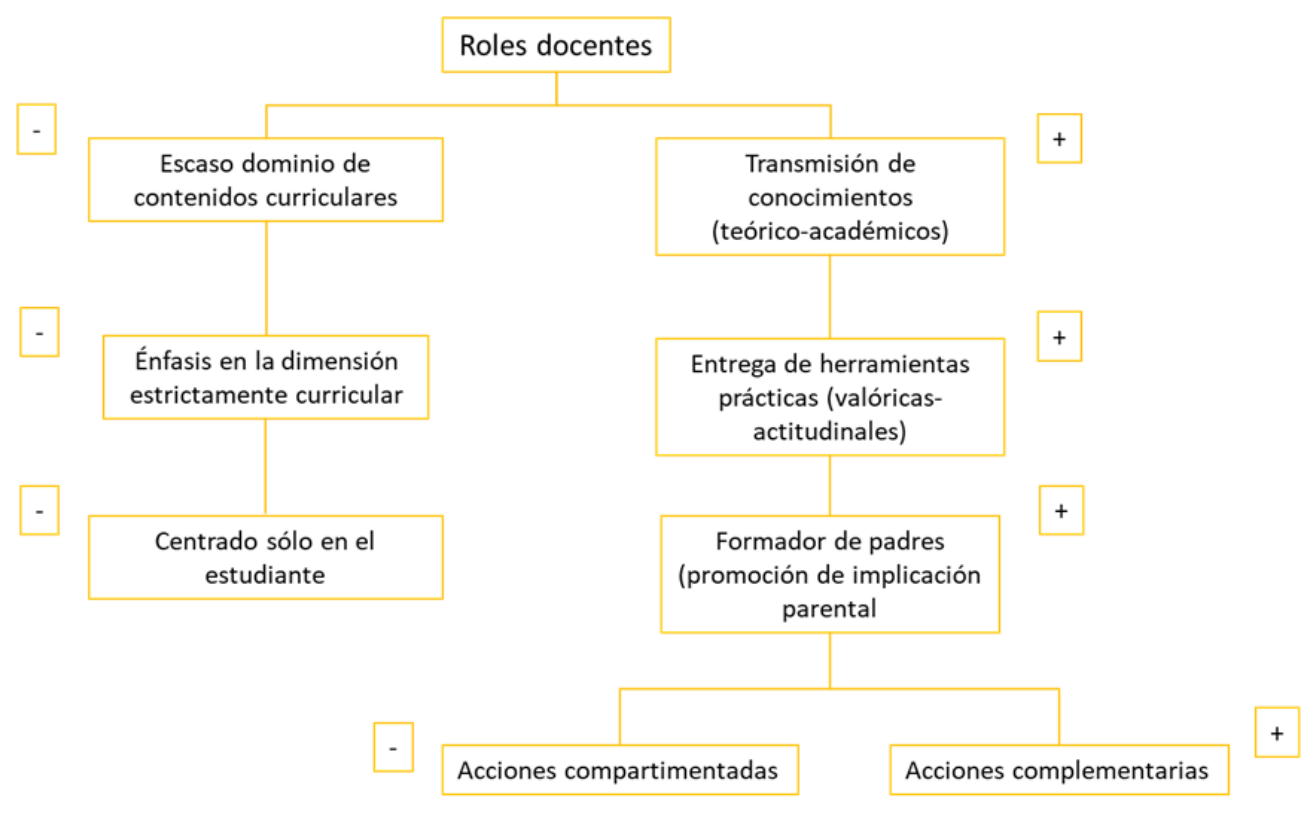

Figura 5. Eje de significación: roles docentes

Los sujetos entrevistados reconocen en las familias al primer agente educativo y, tal como es señalado en la literatura, asumen que la escuela y ellos mismos representan el segundo agente socializador por excelencia. De esta manera, la necesidad de complementar esfuerzos se torna, a juicio de los entrevistados, en tarea fundamental. Así lo refleja Sara:

Las primeras personas que educan están en la familia, el profesor es más que nada el que lo guía, que complementa los valores que le entrega la familia y él, bueno lo va guiando al camino del bien. Le entrega otros conocimientos que son más académicos, que a futuro le van a servir para trabajar, seguir sus estudios, pero ese es el principal trabajo del profesor, es como guiar. (Sara)

La representación de la necesaria complementariedad está mediada por la supremacía del área de influencia de la escuela. Toda vez que se plantea la necesidad de apoyarse mutuamente, aparece la intención de extender los brazos de lo escolar al ámbito familiar. Tan es así, que uno de los roles atribuidos al profesorado es el de formador de padres. ¿Para qué?, para contribuir a un mejor perfil educativo de los niños. Sobre esto, Fernanda plantea:

Ahí es el rol del profesor, enseñarle al papá a cómo ayudar a su hijo. (Fernanda)

Tal como se ha podido apreciar a lo largo de este apartado, la representación del rol docente no sintoniza adecuadamente con lo estipulado en los estándares pedagógicos (MINEDUC, 2012) ni con lo declarado en las bases curriculares para educación básica, situación que pone de manifiesto la necesidad de atender a esta dimensión desde la formación inicial docente con el fin de desnaturalizar el rol escolar centrado que fluye entre los futuros profesores.

\subsection{Acerca de la relación familia escuela}

Los roles que los sujetos asignan a las familias y a sí mismos en tanto que futuros profesores, son elementos constitutivos y constituyentes de las representaciones que poseen respecto de la relación familia-escuela. Se aprecia una tendencia a representar la relación en un estado de situación caracterizado por la fragilidad, producto de lo que consideran una escasa preocupación de los padres por la educación de sus hijos. Esta despreocupación se expresa de manera homogénea, sin considerar diferencias entre las familias según nivel sociocultural, a diferencia de lo expuesto por Domina (2005). Al respecto, Ignacio comenta: 
La participación es escasa, porque como le digo no se introducen mayormente en lo que es la vida estudiantil del alumno, no les preocupa mucho, hoy en día los apoderados van solamente a reclamarle a un profesor. (Ignacio)

Al reconocer un vínculo débil entre las familias y la escuela, los entrevistados lo hacen desde la noción de participación y no de relación. Asumiendo que relación y participación son cosas distintas, se desea destacar que, con independencia de la distinción, los sujetos coinciden en destacar la importancia que reviste el fortalecimiento del vínculo, el mayor involucramiento de los padres. Pero, como se verá más adelante, siempre atendiendo a las demandas emanadas desde el campo escolar. A este respecto, Camila sostiene:

Es que creo que los padres deben involucrarse mucho porque es la educación de sus hijos, pero hay que tener buena comunicación, pero también tiene que haber un, no sé, como un rayado de cancha por así decirlo, en donde el profesor tenga su espacio y que no sea cuestionado por el papá y donde el profesor... donde el papá tenga un espacio y no sea cuestionado por el profesor. (Camila)

Sin importar si se refieren a relación o participación, se aprecia que la representación respecto de la relación familia-escuela se forja sobre la base de tres elementos. El primero muestra que ésta se forja en relación con la adjetivación del tipo de vínculo (débil, frágil). El segundo, sustentado en una orientación moral en torno a la responsabilidad que ha de asumir la familia en la educación de los hijos: deben involucrarse. Tal como se aprecia en el discurso de Camila, este involucramiento implica una forma ajustada a reglas establecidas desde la escuela, citando a Camila: "tiene que haber un rayado de cancha". Esta metáfora denota el interés por resguardar el propio quehacer en tanto que futuros profesores, evitando la intromisión de las familias en el desempeño profesional docente (figura 6). En la medida en que se manifiesta una representación de la participación de las familias como posible intromisión, se forja un posicionamiento que se distancia de lo esperado en los estándares pedagógicos para los egresados de educación básica (MINEDUC, 2012).

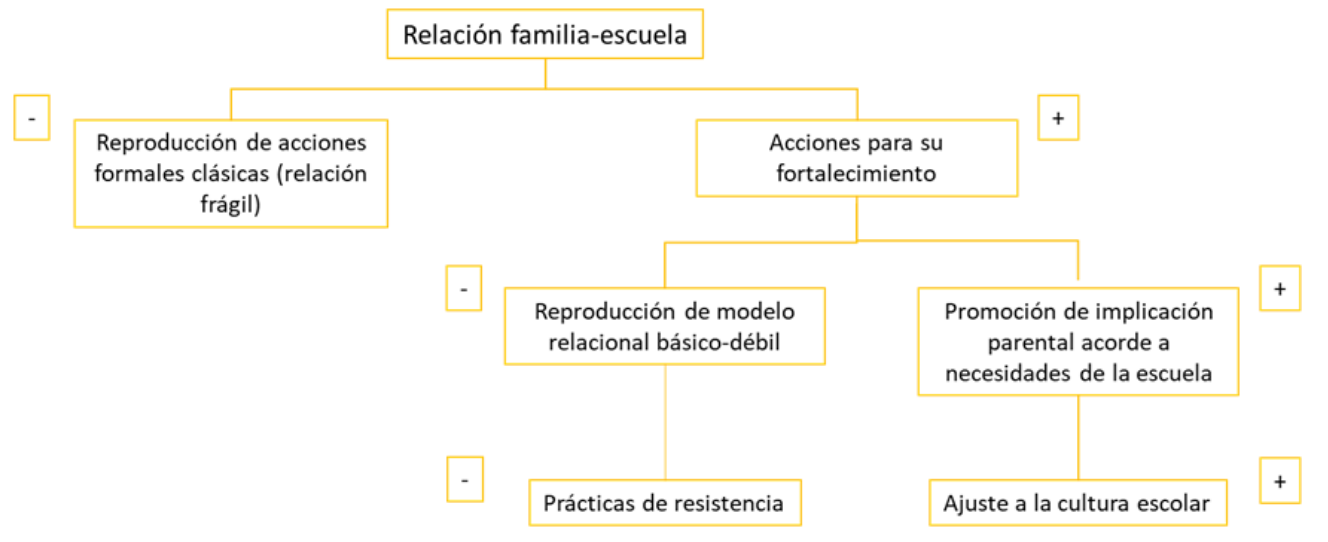

Figura 6. Eje de significación: Relación familia-escuela

El tercer elemento sobre el cual se configura la representación respecto de la relación familia-escuela se desprende de lo expuesto por Esteban, quien alude a la dimensión práctica a través de la cual ha de manifestarse lo que estima como "adecuada relación".

Yo creo que debería ser bastante cercana porque, por ejemplo, a un profesor le ayudaría a entender por qué su hijo reacciona de tal manera frente a alguna situación, que tengamos un contacto con el apoderado, que el apoderado diga "mi hijo tiene tal problema" o "sufrió tantas cosas" entonces, que sepan cómo es el niño, verlo físicamente, saber si es desordenado, estudioso o porfiado. Tener la visión de los padres y ayudarlos para que en el aula dejen esas cosas de lado y puedan ser buenos alumnos. (Esteban) 
La expresión concreta de una adecuada relación se manifiesta toda vez que se generan espacios en que los profesores obtienen insumos -desde las propias familias- para promover actitudes ajustadas a la cultura escolar, actitudes que por cierto faciliten el adecuado desempeño docente dentro del aula. Se evidencia la necesidad de fomentar la escucha activa entre el profesorado como recurso para fortalecer la relación familia-escuela.

\section{Conclusiones}

Un primer elemento que cabe destacar es que se reconoce la relevancia que los sujetos otorgan a la relación familia-escuela, a pesar de la escasa formación que reciben al respecto desde el plan de estudios vigente. Cabe agregar que la relevancia a que aluden los entrevistados va en dirección a la consecución de objetivos de naturaleza escolar, tal como han expuesto algunos autores (Cárcamo-Vásquez y Rodríguez-Garcés, 2015; Palaudàrias, 2017; Pascual y Gomila, 2012). La necesidad de proyectar acciones centradas en el fortalecimiento de la relación entre los agentes se considera fundamental en la medida que contribuya al buen desempeño académico de los niños, así como mientras dichas acciones operen a modo de complemento para cubrir los requerimientos de la escuela y sus docentes, situación que también ha sido evidenciada por autores como Garreta (2017) y Domina (2005). Y en el caso Chileno no es muy diferente, ya que como hemos indicado, la relación y la comunicación entre los profesionales y las familias son consideradas básicas para conseguir los objetivos educativos (Gubbins, 2016) y, de hecho, la administración educativa lo explicita en sus documentos (MINEDUC, 2017).

La representación que poseen los sujetos respecto a la relación entre familias y escuelas está permeada por la experiencia propia en tanto que ex alumnos del nivel básico de educación, pero también por el proceso de formación inicial docente que cursan actualmente. Ambos elementos han de considerarse como fuentes para la configuración de la representación. En ese sentido, pueden visualizarse a familias y docentes como agentes que intervienen en este modelamiento, pues se constituyen en agentes fundamentales en ambos momentos de escolarización (educación básica y educación superior). Pero en el caso de la experiencia vivida a nivel de la formación inicial docente, los pares cobran relevancia en la medida en que es en el diálogo con ellos como se va forjando una imagen del profesor que desean proyectar. Al respecto, instancias formativas, como las prácticas rural y profesional, proporcionan insumos vivenciales de gran valía.

Expuesto lo anterior, la representación familia-escuela deriva de contenidos específicos en torno al concepto de roles. Sobre la base de los elementos de la sociología clásica, se considera que los roles son papeles que los agentes sociales deben desempeñar para mantener el funcionamiento adecuado de las instituciones; en este caso, la escuela. Así, prestan especial atención a los roles que, desde su perspectiva, deben desempeñar tanto las familias como los docentes.

Los roles parentales que los sujetos consideran deben ser desarrollados por las familias se sitúan en dos dimensiones claramente definidas y una tercera que opera como elemento complementario.

Por una parte, los futuros profesores consideran que las familias (desde sus hogares) deben cumplir con un rol parental de apoyo a las actividades desarrolladas en la escuela; en tal sentido, se visualiza una suerte de naturalización de la alumnización de la vida de los niños, pues se plantea como necesaria la proyección del espacio escolar al mundo familiar. De este modo, los contenidos de la información que circula desde la escuela a la familia y las formas de comunicarlos son consecuentes con el objetivo de alumnización referido antes. En tal sentido, se asigna un alto nivel de responsabilidad a las familias para el establecimiento de lo que, en tanto que futuros profesores, consideran debe ser la relación con la escuela, a pesar de la imagen desmejorada que tienen del cumplimiento del rol parental educativo por parte de las familias, situación que coincide con los hallazgos de Pascual y Gomila (2012).

Por otra parte, situándose en extramuros del espacio escolar, los sujetos asignan a las familias el papel de formadores de hábitos, por tanto, se les reconoce el rol de modelador actitudinal (Enkvist, 2011). No se trata de cualquier hábito sino de hábitos que contribuyan a la generación de un clima de aprendizaje que estiman adecuado para la realización de clases y, por consiguiente, un clima de aula que facilite la 
consecución de los objetivos de aprendizaje propuestos desde el espacio escolar.

A modo de complemento, los entrevistados señalan que la participación de las familias en la escuela -ya sea en reuniones grupales o entrevistas individuales convocadas por el docente- es un rol clave a desarrollar por las familias, pero ya no para la consecución de objetivos de aprendizajes sino como indicador de compromiso parental. La asistencia a las instancias mencionadas, pone de manifiesto -según los entrevistados- la disposición que poseen las familias hacia la escucha, así como su voluntad de ser informados del acontecer de la escuela y las demandas del profesorado, resaltando la importancia de la comunicación como objeto de atención para el establecimiento de la relación familia-escuela (Garreta y Maciá, 2017).

Por otra parte, los roles que asignan al profesorado se circunscriben a la arena del proceso de enseñanza y aprendizaje. La transmisión de contenidos curriculares de naturaleza teórico-académica aparece como elemento de gran relevancia, pero los entrevistados reconocen que esta dimensión en sí misma, aunque necesaria, no es suficiente. De este modo, visualizan la pertinencia de articular estos aspectos con otra dimensión que conceptúan como práctica. La entrega de herramientas para transitar exitosamente por el campo escolar y el modelamiento de actitudes acordes con la cultura escolar adquieren un rol protagónico.

Finalmente, cuando los sujetos aluden a la necesidad de fortalecer la relación familia-escuela y la consecuente implicación parental, lo hacen desde una perspectiva escolar-centrada. Puesto que la implicación parental se vuelve un pilar estratégico para la consecución de las metas trazadas desde el espacio burocrático de la escuela, sin problematizar su rol en tanto que futuros profesores a la luz de lo definido en los estándares pedagógicos y las bases curriculares de educación básica vigentes. En el caso chileno parece ser un reto hacer más mención en los documentos de la administración educativa y en la normativa sobre la importancia de potenciar la relación, la comunicación y la implicación de las familias en los centros escolares, hecho que de seguro irá impregnando la formación de los docentes y se irá trasladando a las prácticas en las escuelas y, evidentemente, en las representaciones que tienen los docentes de su propio rol y el de las familias.

\section{Referencias}

Calixto, R. (2015). El cambio climático como objeto de representación. En R. Calixto (Coord.), Representaciones sociales en la práctica educativa y en la formación docente (pp. 35-50). ISCEEM.

Cárcamo, H. (2015). Representaciones sociales sobre el rol del profesorado: miradas desde la formación inicial docente. En R. Calixto (Coord.), Representaciones sociales en la práctica educativa y en la formación docente (pp. 89-106). ISCEEM.

Cárcamo-Vásquez, H. y Rodríguez-Garcés, C. (2015). Rol parental educativo: aproximación a las percepciones que poseen los futuros profesores. Revista Educación y Educadores, 18(3), 456-470. http://doi.org/10.5294/edu.2015.18.3.5

Cerletti, L. B. (2010). Familias y escuelas: aportes de una investigación etnográfica a la problematización de supuestos en torno a las condiciones de escolarización infantil y la categoría familia. Revista Intersecciones en Antropología, 11(1), 185-198. https://www.ridaa.unicen.edu.ar/xmlui/handle/123456789/1148

Coller, X. (2005). Estudio de casos. Cuadernos metodológicos 30. Centro de Investigaciones Sociológicas.

Domina, T. (2005). Leveling the home advantage: assesing the effectiveness of parental involvement in elementary school. Sociology of Education, 78(3), 233-249. https://doi.org/10.1177/003804070507800303

Enkvist, I. (2011). La buena y la mala educación. Encuentro.

Erikson, L. (2004). Föräldrar och skola [Parents and School]. (tesis doctoral). Örebro University.

http://oru.diva-portal.org/smash/record.jsf?pid=diva2\%3A137381\&dswid=3423 
Gaínza, A. (2006). La entrevista en profundidad individual. En M. Canales (Ed.), Metodología de investigación social. Introducción a los oficios (pp. 219-264). LOM.

Garreta, J. (2015). La comunicación familia-escuela en educación infantil y primaria. Revista de la Asociación de Sociología de la Educación, 8(1), 71-85. https://ojs.uv.es/index.php/RASE/article/view/8762

Garreta, J. (2017). La participación de las familias en los centros educativos. En J. Garreta (Coord.), Familias y escuelas. Discursos y prácticas sobre la participación en la escuela (pp.17-26). Pirámide.

Garreta, J. y Maciá, M. (2017). La comunicación familia-escuela. En J. Garreta (Coord.), Familias y escuelas. Discursos y prácticas sobre la participación en la escuela (pp. 71-98). Pirámide.

Grant, B. K. y Ray, J. A. (2013). Home, school and community collaboration: culturally responsive family engagement. Sage.

Gubbins, V. (2014). Estrategias educativas de familias de clase alta. Revista Mexicana de Investigación Educativa, 19(63), 1069-1089.

https://www.comie.org.mx/revista/v2018/rmie/index.php/nrmie/article/view/220

Gubbins, V. (2016). Relación familias y escuelas: ¿por qué y para qué? Universidad Finis Terrae.

Jodelet, D. (1986). La representación social: fenómenos, conceptos y teoría. En S. Moscovici (Ed.), Psicología Social II. Pensamiento y vida social. Psicología social y problemas sociales (469-494). Paidós.

Lacolla, L. (2015). Representaciones sociales de los estudiantes acerca de las reacciones químicas. En R. Calixto (Coord.), Representaciones sociales en la práctica educativa y en la formación docente (pp.19-34). ISCEEM.

Lareau, A. (2002). Invisible inequality: social class and childrearing in black families and White families. American Sociological Review, 67(5), 747-776. https://doi.org/10.2307/3088916

Londoño, L. V. y Ramírez, L. (2012). Construyendo relación familia-escuela: consideraciones a partir de intervención interdisciplinaria en el Colegio Bello Oriente en Medellín, Colombia. Revista Virtual Universidad Católica del Norte, 36, 193-220. https://revistavirtual.ucn.edu.co/index.php/RevistaUCN/article/view/375

Martinic, S. (2006). El estudio de las representaciones y al análisis estructural de discurso. En M. Canales (Ed.), Metodologías de investigación social. Introducción a los oficios (pp. 299-320). LOM.

McWayne, C., Downer J., Campos, R. y Harris, R. (2013). Father involvement during early childhood and its association with children's early learning: a meta-analysis. Early Education y Development, 24, 898-922.

Ministerio de Educacion. (2012). Estándares orientadores para egresados de carreras de pedagogía en educación básica. Autor. https://www.cpeip.cl/wp-content/uploads/2016/07/librobasicaokdos.pdf

MINEDUC (2013). Bases Curriculares para la Educación Básica.

https://www.curriculumnacional.cl/605/articles-30013 recurso 15.pdf

MINEDUC (2017). Política de participación de las familias y la comunidad en instituciones educativas. Autor. https://basica.mineduc.cl/wp-content/uploads/sites/25/2017/04/Pol\%C3\%ADtica-deParticipaci\%C3\%B3n-de-la-Familia-y-la-Comunidad-en-instituciones-educativas.pdf

Moscovici, S. (1979). El psicoanálisis, su imagen y su público. Huemul. 
Palaudàrias, J. M. (2017). Cómo se entiende la participación de las familias en y desde las escuelas. En J. Garreta (Coord.), Familias y escuelas. Discursos y prácticas sobre la participación en la escuela (pp.27-48). Pirámide.

Pascual, B. y Gomila, M. A. (2012). La construcción del discurso social de los futuros docentes en relación a la participación de las familias. Revista Tempora, 15, 93-106. https://riull.ull.es/xmlui/handle/915/4750

Pérez, A. (2010). Aprender a educar. Nuevos desafíos para la formación de docentes. Revista Interuniversitaria de formación del profesorado, 68(24-2), 37-60. file:///C:/Users/Erika/Downloads/DialnetAprenderAEducar-3276044.pdf

Piña, J. (2013). La teoría de las representaciones sociales. Nociones y linderos. En L. Llanos (Ed.), Metodología y enfoques interdisciplinarios de investigación en las ciencias sociales (pp.161-193). Universidad Autónoma Chapingo.

Río, M. A. (2010). No quieren, no saben, no pueden: categorizaciones sobre las familias más alejadas del campo escolar. Revista Española de Sociología, 14, 85-105.

https://recyt.fecyt.es/index.php/res/article/view/65180

Romagnoli, C. y Gallardo, G. (2007). Alianza efectiva familia escuela: para promover el desarrollo intelectual, emocional, social y ético de los estudiantes. Valoras uC.

Saraiva, L. y Wagner, A. (2013). A relação família-escola sob a ótica de professores e pais de crianças que frequentam o ensino fundamental [La relación familia-escuela bajo la óptica de profesores y padres de niños de la educación general básica]. Ensaio: Avaliação e Políticas Públicas em Educação, 21(81), 739-772. https://doi.org/10.1590/S0104-40362013000400006

Schilling, C. (2015). La construcción de nuevas interacciones entre familia y escuela: avanzando hacia una perspectiva comunicativa del espacio escolar. En V. Nogués y A. Precht (Eds.), Nuevas formas de relación en la escuela: reflexionar y transformar (pp.195-214). Universidad Santo Tomás.

Schutz, A. (2003). El problema de la realidad social. Escritos I. Amorrortu.

Segovia, J. D., Martos, M. A. y Martos, L. D. (2010). Colaboración familia-escuela en España: retos y realidades. Revista de Estudios y Experiencias en Edición, 9(18), 111-133.

http://www.rexe.cl/ojournal/index.php/rexe/article/view/133

Toledo, U. (2014). El programa sociofenomenológico de investigación. En F. Osorio (Ed.), Epistemología y ciencias sociales: ensayos latinoamericanos (pp. 39-68). LOM.

Walker, J. M. T. y Hoover-Dempsey, K. V. (2008). Parent involvement. En T. Good (Ed.), 21st Century Education: A reference handbook (382-392). Sage. http://dx.doi.org/10.4135/9781412964012.n90 